\title{
Podoplanin-positive cancer cells at the edge of esophageal squamous cell carcinomas are involved in invasion
}

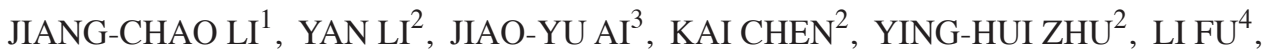 \\ YAN-RU QIN ${ }^{3}$, LI-JING WANG ${ }^{1}$ and XIN-YUAN GUAN ${ }^{2,4}$ \\ ${ }^{1}$ Vascular Biology Research Institute, Guangdong Pharmaceutical University, Guangzhou, Guangdong 510006; \\ ${ }^{2}$ State Key Laboratory of Oncology in Southern China, Sun Yat-Sen University Cancer Center, Guangzhou, \\ Guangdong 510060; ${ }^{3}$ Department of Oncology, The First Affiliated Hospital, Zhengzhou University, \\ Zhengzhou, Henan 450052; ${ }^{4}$ Department of Clinical Oncology, The University of Hong Kong, \\ Pokfulam, Hong Kong, SAR, P.R. China
}

Received October 21, 2013; Accepted April 24, 2014

DOI: $10.3892 / \mathrm{mmr} .2014 .2338$

\begin{abstract}
Podoplanin (PDPN) is a well established lymphatic endothelial marker and has frequently been observed in cancer cells at the edge of cancer masses. Previous studies investigating the association between PDPN expression and patient prognosis have had contradictory results. In the present study, it was hypothesized that the different locations of PDPN-positive cells may explain these varying results. The present study aimed to focus on PDPN expression at the edge of esophageal cancer cell nests. In order to analyze the clinical significance of this PDPN expression, immunohistochemistry was performed using esophageal cancer tissue microarrays. PDPN expression at the edge of the cancer cell nest was found to be significantly associated with invasion $(\mathrm{P}<0.05)$ and poor prognosis $(\mathrm{P}<0.001)$ in patients with cancer. To further investigate the role of PDPN expression in cancer cells, the PDPN gene was cloned and transfected into esophageal squamous cell carcinoma (ESCC) cell lines. PDPN expression was also knocked down using small interfering RNA. PDPN-positive cancer cells were found to exhibit invasion characteristics. Thus, PDPN expression at the edge of a cancer cell nest may indicate invasion and represent a poor prognostic factor for ESCCs.
\end{abstract}

\section{Introduction}

The mucin-type transmembrane glycoprotein podoplanin (PDPN) is a specific marker for lymphatic endothelial cells (1-5) and is frequently used to detect lymphatic numbers

Correspondence to: Professor Xin-Yuan Guan, State Key Laboratory of Oncology in Southern China, Sun Yat-Sen University Cancer Center, 651 Dongfeng Road East, Guangzhou, Guangdong 510060, P.R. China

E-mail: xyguan@hkucc.hku.hk

Key words: podoplanin, esophageal squamous cell carcinoma, invasion, prognosis in various cancer tissues, including colon and breast cancer (6-17). However, PDPN is also present in normal follicles and cancer-associated stromal cells $(18,19)$.

PDPN expression is associated with tumor invasion, metastasis and poor prognosis in cancer cells $(8,15,20,21)$. However, it has also been reported that PDPN inhibits cancer cell invasion (22-25). Furthermore, a recent study found that PDPN-positive lymphatic vessel invasion is not a poor prognostic factor in stage I lung adenocarcinoma (26). In the present study, it was hypothesized that the different locations in which PDPN-positive expression is observed, may explain these contradictory results. In order to determine the function of PDPN at the edge of cancer cell nests, cancer cells expressing PDPN in these specific locations were investigated. Immunohistochemistry was performed using esophageal squamous cell carcinoma (ESCC) tissue microarrays (TMAs; 234 tissues) in order to verify the clinical significance of PDPN expression. Furthermore, the effect of PDPN overexpression and knock down was investigated in cancer cells. PDPN expression was found to be associated with ESCC cancer cell invasion; however, further investigation is required.

\section{Materials and methods}

Clinical samples and cell lines. A total of 234 informative esophageal cancer tissues and 71 non-cancerous esophageal tissues collected between January 2003 and December 2007 were obtained from the archives of the Linzhou People's Hospital (Linzhou, China). All samples were used to produce the TMA sections. The present study was approved by the Committee for the Ethical Review of Research Involving Human Subjects at Zhengzhou University (Zhengzhou, China) and Sun Yat-Sen University (Guangzhou, China). The clinical and pathological information from the patients with ESCC was partially complete. Mean patient age was 60.3 years at the time of surgery. Tumor stage assessment was classified according to the World Health Organization grading criteria and the tumor, nodes and metastasis (TNM) stage was classified according to the sixth version of the Union for International Cancer Control TNM classification criteria. 
Nine lung cancer cell lines were authenticated by testing for mycoplasma contamination using Mycoalert ${ }^{\mathrm{TM}}$ (Lonza Group, Basel, Switzerland) and through analyzing cell morphology. The HKESC1, EC18 and EC109 lung cancer cell lines were provided by Professor Tsao and Professor Srivastava (University of Hong Kong, Hong Kong, China) and the KYSE30, KYSE140, KYSE180, KYSE410, KYSE510 and KYSE520 lung cancer cell lines were obtained from DSMZ (Braunschweig, Germany).

Immunohistochemical analysis of TMAs. The sections were deparaffinized according to routine pathological techniques. Antigen retrieval was performed by boiling the specimens for $15 \mathrm{~min}$ in EDTA solution, then allowing the samples to cool to room temperature. Sections were washed with phosphate-buffered saline (PBS), followed by incubation with anti-human PDPN monoclonal antibodies (Dako UK Ltd, Ely, UK) diluted 1:100. The EnVision ${ }^{\mathrm{TM}}$ detection system (Dako, Kyoto, Japan) was used according to the manufacturer's instructions.

Scoring PDPN-positive cells at the edge of the cancer cell nest. In the present study, tumor sections from the edge of the cancer cell nest which exhibited positive PDPN staining were considered PDPN-positive cases. The immunohistochemical staining score only reflected the distribution of the positive signal at the edge of the cancer cell nest and did not include staining at the center of the cancer cell nest or the stromal or lymphatic regions. Based on the percentage of positive cells at the tumor edge, the samples were given a distribution score as follows: $0,0-5 \% ; 1,6-50 \%$; and 2, 51-100\%. Furthermore, the staining intensity was scored a follows: 0 , no signal; 1 , weak; 2, moderate; and 3, marked. The total score was calculated as the sum of the distribution score and the intensity score and ranged from $0-5$. Total scores between 0 and 1 were considered negative, while scores between 2 and 5 were considered positive.

Plasmid vector construction. HindIII and EcoRI sites (bold) were incorporated into the primer sequences, which were used to amplify the human PDPN gene. The primer sequences were as follows: Forward, 5'-CCCAAGCTTGCCTCCTCGGGA GAGATAAAT G-3' and reverse, 5'-CCGGAATTCAACCCT TCAGCTCTTTAGGGCGA-3'. RNA was obtained from normal placenta tissue and the polymerase chain reaction (PCR) products were inserted into the pcDNA 3.1 plasmid vector (Invitrogen Life Technologies, Carlsbad, CA, USA) according to the manufacturer's instructions. The vector was then sequenced to verify the correct insert.

Quantitative (q)PCR analysis. Total RNA was extracted from the cultured cell lines. The qPCR primer sequences for PDPN were as follows: Forward, 5'-GTGTAACAGGCATTCGCA TCG-3' and reverse, 5'-TGTGGCGCTTGGACTTTGT-3'. PDPN expression was normalized to that of GAPDH and expressed as the fold change relative to the mean level of the control group.

Stable transfection and small interfering (si)RNA knockdown. The pcDNA 3.1 vector containing the human PDPN cDNA insert or the empty pcDNA 3.1 control vector were trans- fected into a series of cell lines using Lipofectamine ${ }^{\circledR} 2000$ (Invitrogen Life Technologies) according to the manufacturer's instructions. Forty-eight hours after transfection, the culture medium was replaced with medium containing $450 \mu \mathrm{g} / \mathrm{ml}$ G418. Cells were then maintained in Dulbecco's modified Eagle's medium (DMEM) containing 10\% fetal bovine serum (FBS) and G418 (Invitrogen Life Technologies) until the resistant cells had grown. Human PDPN siRNA and control siRNA were purchased from Shanghai Genepharma Co., Ltd. (Shanghai, China) and transfection was performed using Lipofectamine 2000.

Proliferation assay. An XTT cell proliferation assay kit (Roche, Basel, Switzerland) was used to determine the proliferation rates of a series of cancer and control cell lines. Cells were seeded at a density of $1 \times 10^{3}$ cells/well on 96-well plates and cultured for five days ( $\mathrm{n}=4$ per cell line).

Invasion assay. Cell invasion was determined using a Transwell ${ }^{\circledR}$ system (BD Biosciences, San Jose, CA, USA) with an $8-\mu \mathrm{m}$ polyethylene terephthalate membrane. The upper chamber was coated with Matrigel ${ }^{\mathrm{TM}}$ and seeded with $1 \times 10^{5}$ cells in DMEM containing $2 \%$ FBS. DMEM containing $10 \%$ FBS was added to the lower chamber. After $18 \mathrm{~h}$, the cells were scraped from the upper side of the membrane using a cotton swab. Subsequent to washing with PBS, the cells that had migrated to the lower chamber were fixed with $75 \%$ ethanol and stained with a $1 \%$ crystal violet solution. The membrane was carefully removed using a knife and mounted onto a glass slide using a sealing reagent. The migrated cells were counted in fields and the mean number of cells counted in five fields was used for the analysis.

Statistical analysis. SPSS 16.0 (SPSS, Inc., Chicago, IL, USA) was used to analyze the data, which are presented as the mean \pm standard deviation. Fisher's exact test or the $\chi^{2}$ test were used to compare the statistical significance of the differences in PDPN expression between the ESCC TMAs. Clinical pathological factors, including age, gender, histological and pathological stages, invasion and TNM stage were considered, and a Log-rank test for survival was performed to compare the positive and negative staining results. Kaplan-Meier curves were plotted according to overall survival. $\mathrm{P}<0.05$ was considered to indicate a statistically significant difference.

\section{Results}

PDPN is frequently expressed at the edge of ESCCs. PDPN has previously been reported to be a lymphatic marker expressed in endothelial cells. The findings of the present study are consistent with those of a previous study, with regard to PDPN expression in patients with ESCC (Fig. 1A) (27). In addition, PDPN was found to be expressed at the edge of the cancer cell nest (Fig. 1B). In order to further verify PDPN cancer cell expression, PDPN mRNA levels were analyzed in various cell lines using qPCR analysis. A total of $66.7 \%$ (6/9) of the ESCC cell lines were observed to express PDPN (Fig. 1C), with the HKESC1, EC18, KYSE140, KYSE180, KYSE510 and KYSE520 cell lines demonstrating PDPN expression, while the EC109, KYSE30 and KYSE410 cell lines did not. 
A

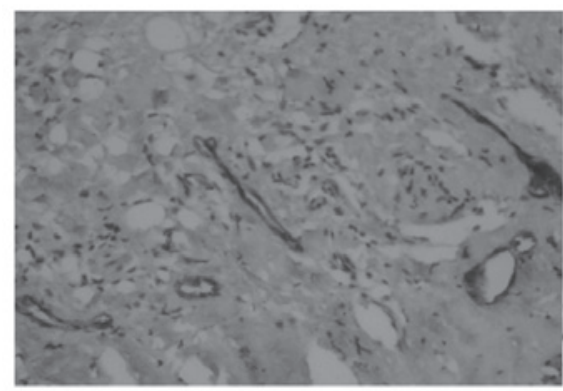

B

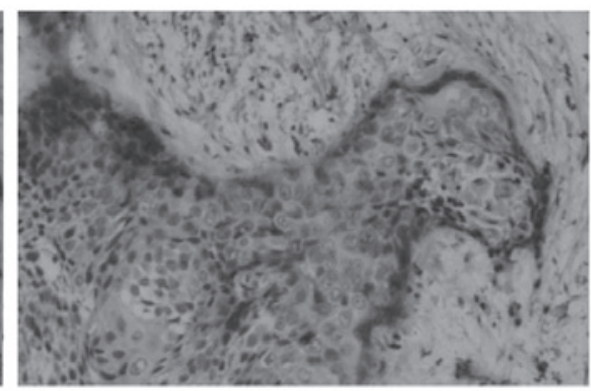

C

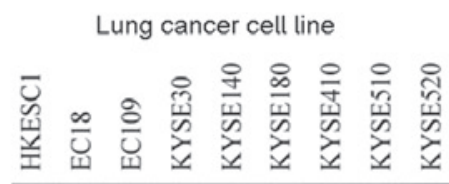

PDPN

GAPDH

Figure 1. PDPN overexpression in ESCC cell lines at the front edge of ESCC tissues. PDPN expression in (A) a lymphatic vessel and (B) at the edge of a cancer cell nest (magnification, x200). (C) PDPN expression was determined using quantitative polymerase chain reaction analysis in nine ESCC cell lines with 67\% (6/9) of the cell lines found to be positive for PDPN expression. PDPN, podoplanin; ESCC, esophageal squamous cell carcinoma.

A

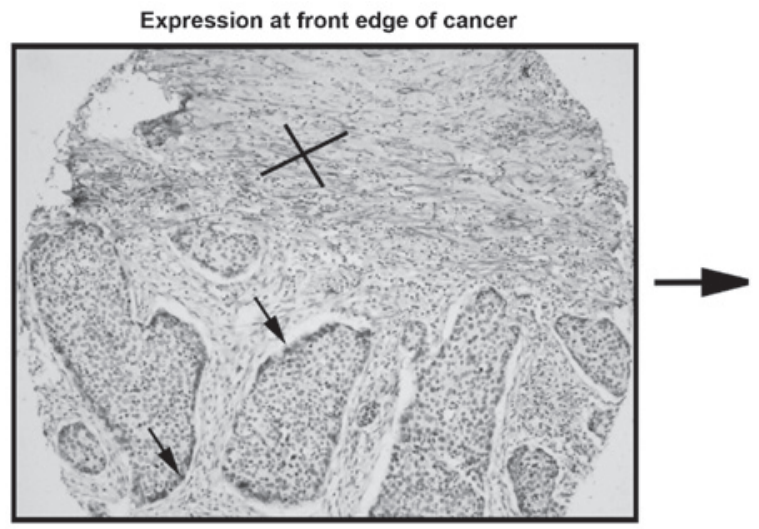

B

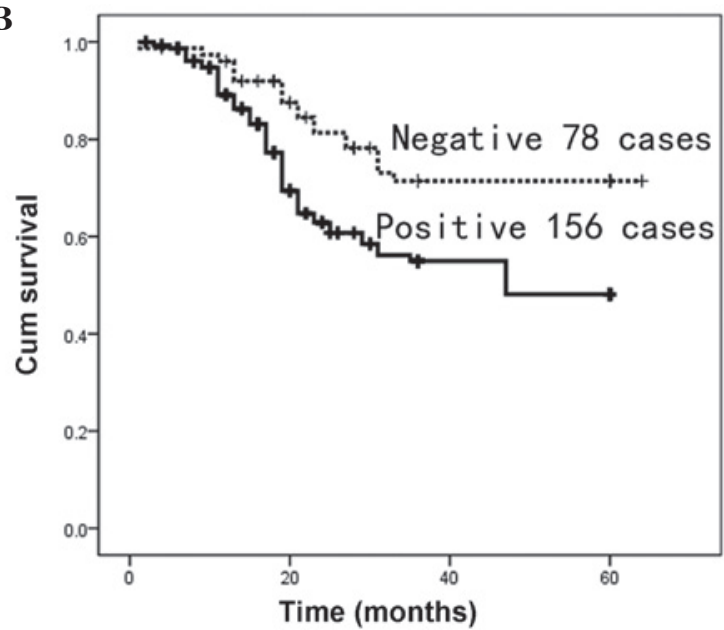

Figure 2. Clinical significance of PDPN expression at the edge of ESCCs in patient samples. (A) PDPN-positive cells at the edge of a cancer cell nest detected using immunohistochemisty of ESCC tissue microarrays (magnification, x100). (B) Survival analysis of patients with PDPN-positive and-negative cells at the edge of their cancer nest. PDPN expression was positive in 66.7\% (156/234) of patients. Kaplan-Meier analysis revealed that the PDPN-positive patient group had a significantly lower overall survival compared with the PDPN-negative group $(\mathrm{P}<0.05)$. PDPN, podoplanin; ESCC, esophageal squamous cell carcinoma.

PDPN expression at the front edge of the cancer cell nest is associated with invasion and poor prognosis. To investigate the clinical significance of PDPN expression at the edge of the ESCC cancer cell nest, the cancer cells from 234 patients with informative clinical and pathological data were investigated. PDPN-positive cells at the edge of the tumor are indicated by the arrow (Fig. 2A). Kaplan-Meier analysis revealed that PDPN cancer cell expression was associated with poor overall survival ( $\mathrm{P}<0.001$; Fig. 2B).

To further assess the significance of PDPN expression in ESCC tissue, the correlation between PDPN expression at the edge of the cancer cell nest and pathological data was assessed in the patients. PDPN expression at the edge of the cancer cell nest was found to be positively correlated with cancer invasion in ESCC tissue (Table I; $\mathrm{P}<0.05$ ), but was not found to be associated with any other clinical features.

PDPN induces invasion, but does not affect growth in ESCC cells. In order to investigate the invasive function of PDPN in ESCC pathogenesis, PDPN was cloned into the pcDNA 3.1 vector and an empty vector was used as the control. The vectors were stably transfected into the KYSE410 and EC109 cell lines and two clones were selected to avoid clone deviation. Prior to the functional analyses, the overexpression of PDPN was determined using qPCR analysis, which revealed increased PDPN expression in the PDPN-transfected cells (Fig. 3A). The effect of PDPN on tumor invasion was then assessed using a Transwell assay. PDPN was observed to significantly promote invasion in the ESCC cell lines ( $\mathrm{P}<0.05$; Fig. $3 \mathrm{C}$ and $\mathrm{D})$.

In addition to the invasion assay, an MTT assay was performed to assess the growth of the KYSE410 and EC109 cells over five days. No significant difference was found in the growth rate of the PDPN-transfected cells compared with the growth rate of the cells expressing the empty vector $(\mathrm{P}>0.05$; Fig. 3B).

PDPN downregulation inhibits tumor invasion. To further analyze whether PDPN has a role in activating cell invasion, PDPN knockdown was performed using an antisense oligonucleotide (Fig. 4A). Wound healing assays revealed that PDPN knockdown markedly inhibited KYSE520 cell motility compared with that of the control cells (Fig. 4B). To assess the invasive capacity of the PDPN knockdown cell lines, a cell invasion assay was performed using a coated Matrigel Transwell system. Statistical analysis demonstrated that PDPN silencing significantly inhibited invasion in the tumor cells. 
Table I. Clinical signficance of PDPN expression at the front edge of the cancer cell nest in 234 primary ESCCs.

\begin{tabular}{|c|c|c|c|c|}
\hline \multirow[b]{2}{*}{ Clinical parameter } & \multirow[b]{2}{*}{ Cases (n) } & \multicolumn{2}{|c|}{ PDPN expression, $\mathrm{n}(\%)$} & \multirow[b]{2}{*}{ P-value } \\
\hline & & Positive & Negative & \\
\hline Gender & & & & 0.229 \\
\hline Female & 116 & $73(62.9)$ & $43(37.1)$ & \\
\hline Male & 118 & $83(70.3)$ & $35(29.7)$ & \\
\hline Age & & & & 0.141 \\
\hline$\leq 60$ & 134 & $85(63.4)$ & $49(36.6)$ & \\
\hline$>60$ & 100 & $71(71.0)$ & $29(29.0)$ & \\
\hline Lymph node metastasis & & & & 0.888 \\
\hline N0 & 135 & $89(71.2)$ & $46(36.8)$ & \\
\hline N1 & 99 & $67(67.6)$ & $32(32.3)$ & \\
\hline TNM stage & & & & 1.00 \\
\hline Early stage (I-II) & 159 & $106(66.7)$ & $53(33.3)$ & \\
\hline Advanced stage (III-IV) & 75 & $50(66.7)$ & $25(33.3)$ & \\
\hline Tumor size $\left(\mathrm{cm}^{3}\right)$ & & & & 0.166 \\
\hline$\leq 5$ & 29 & $13(44.8)$ & $16(51.2)$ & \\
\hline$>5$ & 148 & $87(50.6)$ & $61(49.4)$ & \\
\hline Distance metastasis & & & & 0.858 \\
\hline Metastasis & 4 & $2(50.0)$ & $2(50)$ & \\
\hline No metastasis & 230 & $154(67.0)$ & $76(33.0)$ & \\
\hline Tumor differentiation & & & & 0.222 \\
\hline Good (I-II) & 29 & $19(65.5)$ & $10(34.5)$ & \\
\hline Moderate (III) & 155 & $103(66.5)$ & $52(33.5)$ & \\
\hline Poor (IV) & 48 & $34(70.8)$ & $14(29.2)$ & \\
\hline Tumor invasion & & & & 0.013 \\
\hline $\mathrm{T} 1$ & 88 & $50(56.8)$ & $38(43.2)$ & \\
\hline $\mathrm{T} 2$ & 146 & $106(72.6)$ & $40(27.4)$ & \\
\hline
\end{tabular}

Tumor size was measured using the following formula: $0.5 \mathrm{x}$ length $\mathrm{x}$ (width) ${ }^{2}$. Partial data are not available for tumor size and tumor differentiation and the statistic was based on the informative data. Invasion was defined by the results of a final pathological analysis. PDPN, podoplanin; ESCC, esophageal squamous cell carcinoma.

Furthermore, PDPN knockdown was found to induce a more static state compared with the control cells (data not shown).

\section{Discussion}

PDPN expression in lymphatic vessels may have value as a predictive marker of survival. However, PDPN is also expressed in cancer cells, cancer stromal cells and cancer cells at the edge of cancer cell nests. Reports regarding the clinical prognostic significance of PDPN expression in cancer tissues are contradictory, thus further investigation is required. In the present study, it was hypothesized that determining the different locations of PDPN expression may explain the contradictory results.

A previous study by our group found that PDPN was expressed in lymphatic vessels, which was consistent with other reports (Fig. 1A) (28-30). The present study focused on PDPN expression at the edge of cancer cell nests as epithelial-mesenchymal transition is not the only invasion mechanism used by cancerous cells. Preliminary results revealed that PDPN expression at the edge of cancer cell nests was positively correlated with invasion and poor patient survival. These findings indicated that other factors may contribute to cancer cell invasion, as the PDPN-positive cells were invasive without epithelial-mesenchymal transition (22).

The present study showed that PDPN-positive cancer cells have functions which are associated with invasion. Recent reports have shown that PDPN is harmful to patients with ESCC $(27,28)$. Although these studies did not focus on front edge cancer cells, their findings are consistent with those of the present study. The mechanism through which PDPN expression at the edge of a cancer cell nest contributes to cancer cell invasion requires further investigation. It is possible that these cells are induced to express PDPN through factors in the cancer microenvironment; however, the findings of the present study suggested that PDPN has a role in invasion. Tumors are a complex mixture of cells and extracellular matrix (31); therefore, the cross-talk between PDPN-positive cancer cells and the cancer stroma should be investigated. 
A

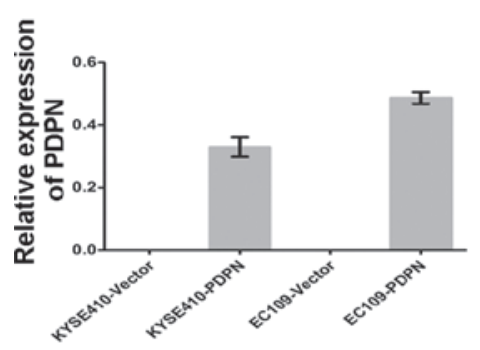

B

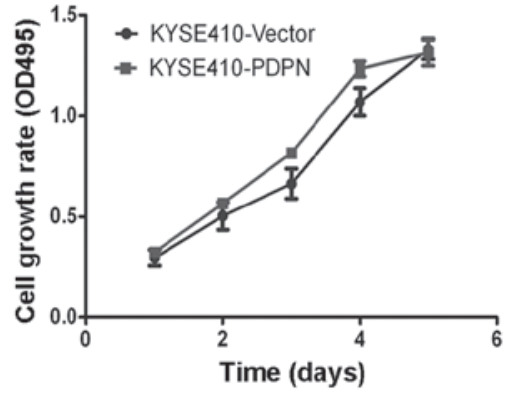

D
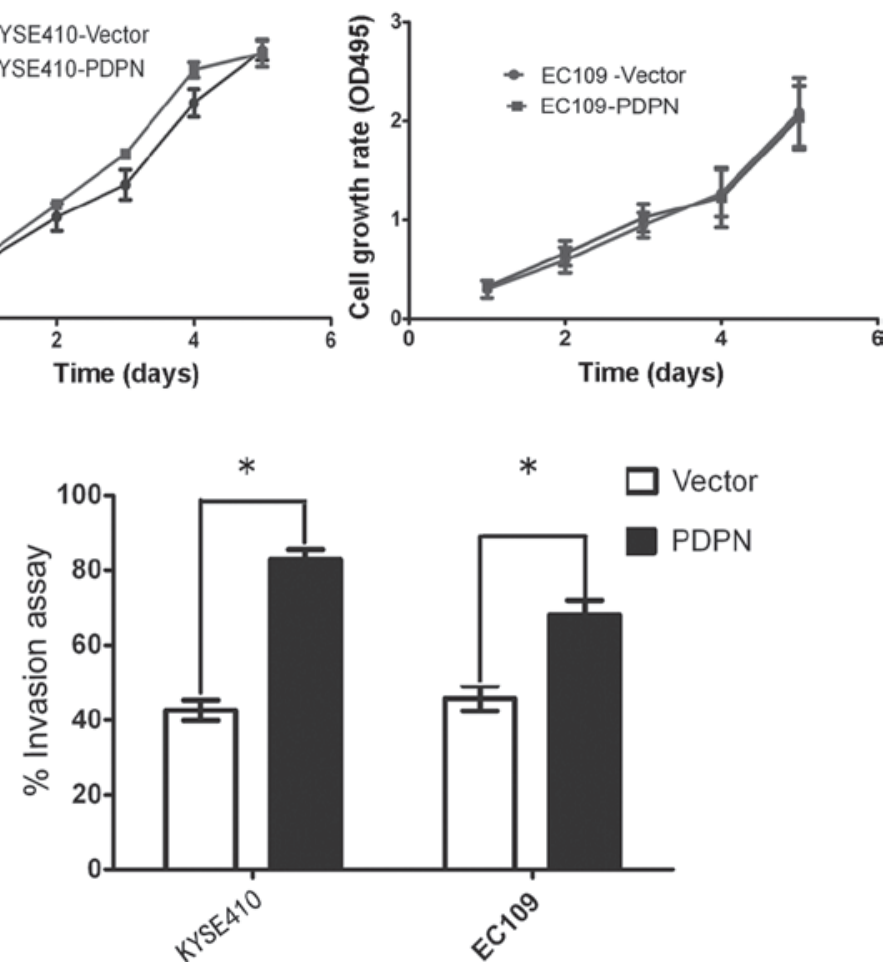

Figure 3. PDPN activates ESCC invasion, but does not affect tumor cell growth. (A) Quantitative polymerase chain reaction analysis revealed increased PDPN expression in PDPN-transfected cells. (B) Cancer cell growth rate in cells transfected with PDPN compared with those transfected with an empty vector detected using MTT assay. Data are presented as the mean \pm standard deviation of three independent experiments. (C) PDPN promoted KYSE410 (upper panels) and EC109 (lower panels) cell migration (magnification, $\mathrm{x} 400$ ). ${ }^{*} \mathrm{P}<0.001$. (D) Results of the invasion assay. ${ }^{*} \mathrm{P}<0.05$. Data are presented as the mean \pm standard deviation of three independent experiments. PDPN, podoplanin.

A

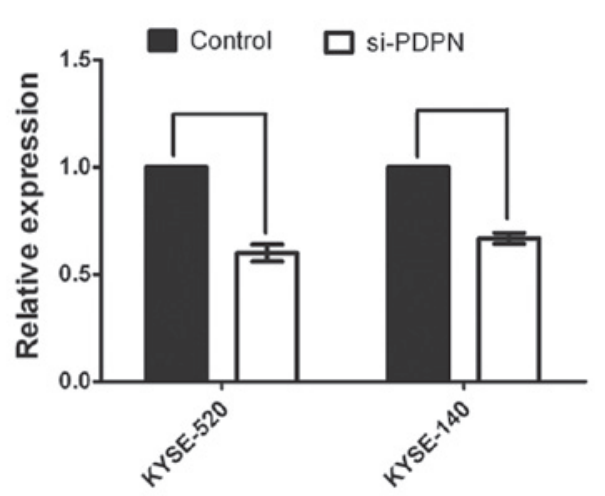

C

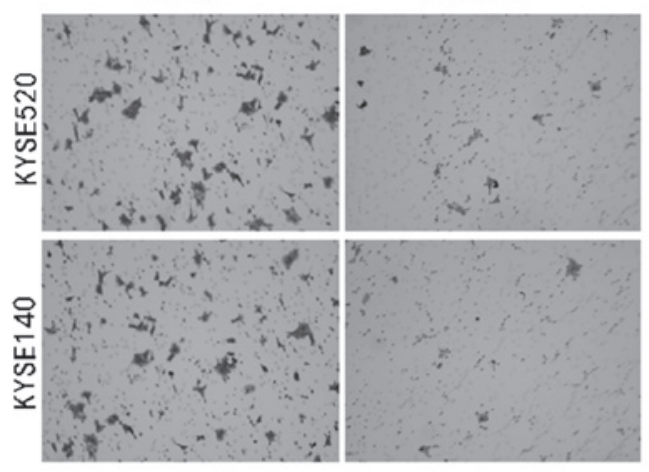

B

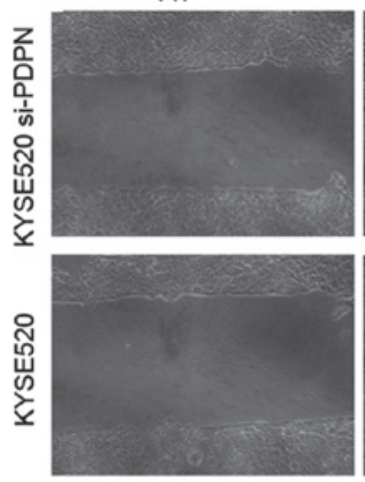

D

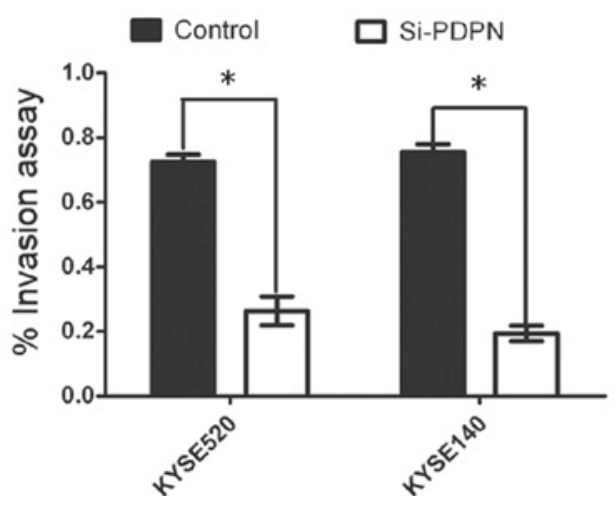

Figure 4. PDPN knockdown reduces tumor cell migration and invasion. (A) PDPN was silenced through transfection with siRNA. PDPN knockdown was confirmed using quantitative polymerase chain reaction analysis in the KYSE520 and KYSE140 cell lines. (B) Tumor cell motility was reduced following PDPN knockdown. Representative images of KYSE520 were captured at 0 and 48 h. (C) Cancer cell invasion was determined using a Transwell ${ }^{\mathrm{TM}}$ assay. PDPN knockdown reduced cell migration (magnification, $\mathrm{x} 400$ ) (D) Cell invasion is expressed as the mean \pm standard deviation of three independent experiments. "P<0.05. PDPN, podoplanin; si, small interfering. 
Future studies by our group will focus on investigating the invasion mechanism induced by PDPN expression. Understanding the role of PDPN in cancer cells will enhance the knowledge of cancer invasion and may ultimately allow researchers to diagnose pathology through targeting PDPN-positive cancer cells at the edge of cancer cell nests. However, more research is still required to achieve these goals.

\section{Acknowledgements}

The present study was supported by grants from the National Natural Science Foundation of China (nos.30772475, 30700820 and 30971606), the Sun Yat-Sen University 'Hundred Talents Program' (no. 85000-3171311) and the Nation Key Sci-Tech Special Project of China (no. 2008ZX10002-022).

\section{References}

1. Kono T, Shimoda M, Takahashi M, et al: Immunohistochemical detection of the lymphatic marker podoplanin in diverse types of human cancer cells using a novel antibody. Int J Oncol 31: 501-508, 2007

2. Yan G, Zhou XY, Cai SJ, et al: Lymphangiogenic and angiogenic microvessel density in human primary sporadic colorectal carcinoma. World J Gastroenterol 14: 101-107, 2008.

3. Moreira LR, Schenka AA, Latuf-Filho P, et al: Immunohistochemical analysis of vascular density and area in colorectal carcinoma using different markers and comparison with clinicopathologic prognostic factors. Tumour Biol 32: 527-534, 2011

4. Ozcelik O, Haytac MC, Ergin M, Antmen B and Seydaoglu G: The immunohistochemical analysis of vascular endothelial growth factors $\mathrm{A}$ and $\mathrm{C}$ and microvessel density in gingival tissues of systemic sclerosis patients: their possible effects on gingival inflammation. Oral Surg Oral Med Oral Pathol Oral Radiol Endod 105: 481-485, 2008.

5. Lee HW, Qin YX, Kim YM, et al: Expression of lymphatic endothelium-specific hyaluronan receptor LYVE-1 in the developing mouse kidney. Cell Tissue Res 343: 429-444, 2011.

6. Kato Y, Kaneko MK, Kuno A, et al: Inhibition of tumor cell-induced platelet aggregation using a novel anti-podoplanin antibody reacting with its platelet-aggregation-stimulating domain. Biochem Biophys Res Commun 349: 1301-1307, 2006.

7. Kato Y, Kaneko M, Sata M, Fujita N, Tsuruo T and Osawa M: Enhanced expression of Aggrus (T1alpha/podoplanin), a platelet-aggregation-inducing factor in lung squamous cell carcinoma. Tumour Biol 26: 195-200, 2005.

8. Yuan P, Temam S, El-Naggar A, et al: Overexpression of podoplanin in oral cancer and its association with poor clinical outcome. Cancer 107: 563-569, 2006.

9. Kimura N and Kimura I: Podoplanin as a marker for mesothelioma. Pathol Int 55: 83-86, 2005.

10. Ordóñez NG: D2-40 and podoplanin are highly specific and sensitive immunohistochemical markers of epithelioid malignant mesothelioma. Hum Pathol 36: 372-380, 2005.

11. Breiteneder-Geleff S, Soleiman A, Kowalski H, et al: Angiosarcomas express mixed endothelial phenotypes of blood and lymphatic capillaries: podoplanin as a specific marker for lymphatic endothelium. Am J Pathol 154: 385-394, 1999.

12. Roy S, Chu A, Trojanowski JQ and Zhang PJ: D2-40, a novel monoclonal antibody against the M2A antigen as a marker to distinguish hemangioblastomas from renal cell carcinomas. Acta Neuropathol 109: 497-502, 2005.
13. Shibahara J, Kashima T, Kikuchi Y, Kunita A and Fukayama M: Podoplanin is expressed in subsets of tumors of the central nervous system. Virchows Arch 448: 493-499, 2006.

14. Mishima K, Kato Y, Kaneko MK, et al: Podoplanin expression in primary central nervous system germ cell tumors: a useful histological marker for the diagnosis of germinoma. Acta Neuropathol 111: 563-568, 2006.

15. Mishima K, Kato Y, Kaneko MK, et al: Increased expression of podoplanin in malignant astrocytic tumors as a novel molecular marker of malignant progression. Acta Neuropathol 111: 483-488, 2006.

16. Fohn LE, Rodriguez A, Kelley MC, et al: D2-40 lymphatic marker for detecting lymphatic invasion in thin to intermediate thickness melanomas: association with sentinel lymph node status and prognostic value-a retrospective case study. J Am Acad Dermatol 64: 336-345, 2011.

17. Grimaldo S, Garcia M, Zhang H and Chen L: Specific role of lymphatic marker podoplanin in retinal pigment epithelial cells. Lymphology 43: 128-134, 2010.

18. Schacht V, Dadras SS, Johnson LA, et al: Up-regulation of the lymphatic marker podoplanin, a mucin-type transmembrane glycoprotein, in human squamous cell carcinomas and germ cell tumors. Am J Pathol 166: 913-921, 2005.

19. Kitano H, Kageyama S, Hewitt SM, et al: Podoplanin expression in cancerous stroma induces lymphangiogenesis and predicts lymphatic spread and patient survival. Arch Pathol Lab Med 134: 1520-1527, 2010.

20. Wicki A,Lehembre F, Wick N, et al: Tumor invasion in the absence of epithelial-mesenchymal transition: podoplanin-mediated remodeling of the actin cytoskeleton. Cancer Cell 9: 261-272, 2006.

21. Gurleyik G, Gurleyik E, Aker F, et al: Lymphovascular invasion, as a prognostic marker in patients with invasive breast cancer. Acta Chir Belg 107: 284-287, 2007.

22. Carvalho FM, Zaganelli FL, Almeida BG, et al: Prognostic value of podoplanin expression in intratumoral stroma and neoplastic cells of uterine cervical carcinomas. Clinics (Sao Paulo) 65: 1279-1283, 2010

23. Kreppel M, Krakowezki A, Kreppel B, et al: Podoplanin expression in cutaneous head and neck squamous cell carcinoma-prognostic value and clinicopathologic implications. J Surg Oncol 107: 376-383, 2013.

24. Suzuki H, Onimaru M, Koga T, et al: High podoplanin expression in cancer cells predicts lower incidence of nodal metastasis in patients with lung squamous cell carcinoma. Pathol Res Pract 207: 111-115, 2011.

25. Suzuki H, Onimaru M, Yonemitsu Y, et al: Podoplanin in cancer cells is experimentally able to attenuate prolymphangiogenic and lymphogenous metastatic potentials of lung squamoid cancer cells. Mol Cancer 9: 287, 2010.

26. Shimizu K, Funai K, Sugimura H, et al: D2-40-positive lymphatic vessel invasion is not a poor prognostic factor in stage I lung adenocarcinoma. Pathol Int 63: 201-205, 2013.

27. Rahadiani N, Ikeda J, Makino T, et al: Tumorigenic role of podoplanin in esophageal squamous-cell carcinoma. Ann Surg Oncol 17: 1311-1323, 2010.

28. Tong L, Yuan S, Feng F and Zhang H: Role of podoplanin expression in esophageal squamous cell carcinoma: a retrospective study. Dis Esophagus 25: 72-80, 2012.

29. Rudno-Rudzinska J, Kielan W, Grzebieniak Z, et al: High density of peritumoral lymphatic vessels measured by D2-40/podoplanin and LYVE-1 expression in gastric cancer patients: an excellent prognostic indicator or a false friend? Gastric Cancer 16: 513-520, 2013.

30. Herzog BH, Fu J, Wilson SJ, et al: Podoplanin maintains high endothelial venule integrity by interacting with platelet CLEC-2. Nature 502: 105-109, 2013.

31. Direkze NC and Alison MR: Bone marrow and tumour stroma: an intimate relationship. Hematol Oncol 24: 189-195, 2006. 